\title{
Proper Planning of Diet is Essential in the Management of Diabetes
}

\author{
Gopal Krushna Pal ${ }^{1, *}$, Nivedita Nanda ${ }^{2}$
}

\section{INTRODUCTION}

India is presently the epicentre of diabetes, hypertension and heart disease. It is mainly the abrupt change in lifestyle, especially the dietary habits and excessive calorie consumption in recent times that has made the population diabetic. Hence, the successful management of diabetes depends on convincing the person to have a proper planning and control of good dietary habits. ${ }^{[1]}$ Diabetes mellitus is a metabolic disease where body can properly neither use nor store energy. When one eats excess calories body responds by creating an undesirable rise in blood glucose. This can lead to hyperglycemia and associated micro- and macro vascular complications. Medical nutrition therapy or MNT is an integral part of diabetes prevention and management. One of its goals is to prevent or manage diabetes by promoting healthy food choices. ${ }^{[2]}$

\section{PLANNING PROTOCOL}

For diabetes prevention as well as management, few tips have to be followed for dietary planning, such as: ${ }^{[3]}$

- $\quad$ Eating frequently but at regular interval: helps our body to keep sugar level under control. As per the Canada's Food Guide one must have at least three out of the four key food groups at each meal from a successful diet plan.

\section{Gopal Krushna Pal',*, Nivedita Nanda²}

'Department of Physiology, JIPMER, Puducherry, INDIA.

${ }^{2}$ Department of Biochemistry, JIPMER, Puducherry, INDIA.

\section{Correspondence \\ Dr. Gopal Krushna Pal}

Department of Physiology, JIPMER, Puducherry, INDIA.

Phone: +919344291160

Email: drgkpal@gmail.com

History

- Received: 18-08-2018

- Revised: 29-09-2018;

- Accepted: 05-10-2018.

DOI : 10.5530/ijcep.2018.5.3.1

\section{Copyright}

(C) 2018 Phcog.Net. This is an openaccess article distributed under the terms of the Creative Commons Attribution 4.0 International license.

- Taking healthy snacks while limiting the intake of sugar, sweets, deserts etc: this avoids sudden spike in blood glucose level.

- $\quad$ Restricting the intake of high fat food such as pastries, chips etc: prevents weight gain due to calorie excess. Avoid saturated fat, Trans fat and cholesterol rich food.

- $\quad$ Avoid processed food such as snacks, baked goods, as they are rich in trans-fat but high in sodium content which increases risk of cardiovascular disease and hypertension respectively.

- Choose lean meat: opt for meat without skin. Fish is a good alternative to high-fat meats

- Limiting alcohol intake: prevents weight gain, calorie build up and protects liver.

- Increasing fiber in diet: promotes satiety, decreases sharp rise in blood glucose level, aids in digestion, prevents cholesterol absorption etc. also helps in prevention of constipation.
- Drinking sufficient water: prevents dehydration and electrolyte imbalance at cellular level. People often mistake thirst for hunger, which can lead to overeating and weight gain

- $\quad$ Try to prepare more of your meals at home and use whole, unprocessed ingredients.

- $\quad$ Try eating together in a family as very often this serves as a model healthy eating behaviours to children and teenagers. ${ }^{[4]}$

With a proper meal plan making its items variable every day while keeping the proximate principles constant is the key for a successful diet plan for diabetes mellitus. The proximate principle in food is nothing but protein, fat, carbohydrate, minerals and water and it's found naturally in plant and animal tissues. Few points have to be kept in mind while planning a diet, such as: ${ }^{[5]}$

a). Counting total calorie: as the final aim of a diet plan is to reduce calorie without compromising nutrients intake, the total calorie intake for each meal a day has to be kept in mind.

b). Counting glycemic index: This method ranks carbohydrate-containing foods based on their effect on blood glucose levels. Examples are legumes, vegetables etc. c). Counting carbohydrate: as carbohydrate directly converts to glucose in blood after metabolism they contribute to hyperglycemia directly. Therefore, one has to eat same amount of carbohydrates daily, at regular intervals, especially if you one is on insulin. As per the Diabetes Canada individuals with diabetes should consult registered dietitian to learn counting carbohydrates in their diet because the carbohydrate content taken at one time is important in managing diabetes.

d). Counting fat: better to avoid high-fat dairy products and animal proteins such as beef, sausage and bacon as they are rich in saturated fats. Opt for fatty food rich in omega-3 fatty acids, mono unsaturated fatty acid in moderation.

e). Mind your portion size: by limiting the portion sizes it helps you to maintain a healthy body weight without the feeling of craving. 


\section{METHODS OF DIET PLANNING ${ }^{[3]}$}

\section{Space Meals}

These are another concept in the planning of diet in diabetes. Here meals are spaced with no more than 6 hours apart. One has to include a variety of food-items per plate such as protein, whole grain, low fat and carbohydrate with lots of fiber in the form of fruits and vegetables. Whole grain examples are brown bread, bran cereals, brown rice etc.

\section{Seven Step Method or Plate Method}

This method of plate filling is has been also advocated by the American Diabetes Association. ${ }^{[3]}$ They describe it as seven step method of planning. This method of plating focuses on eating more of non-starchy vegetables, filling one-half of plate. Examples are spinach, carrots and tomatoes etc. Next one-quarter has to be filled with a protein and last quarter with a whole-grain item or starchy food. Fish such as salmon, mackerel, tuna, sardines and bluefish are rich in omega-3 fatty acids, which promote heart health by lowering blood fats called triglycerides. While fish is a healthy option for non-vegetarians, vegetarians can have a lot of plant protein options such as vegetable protein, such as beans, lentils or tofu etc. One serving of fruit or dairy and a drink of water or unsweetened tea or coffee is advised along with it.

\section{The Diabetic Exchange Lists System}

This serves the purpose of dividing food into six groups. As one goes through the exchange lists, one notices that one choice is often a larger amount of food than another choice from the same list. As one has to combine items from all lists finally the proportion of proximate principles remains the same despite varying/exchanging the items. These are Starch/Bread List, Meat List, Vegetable List, Fruit List, Milk List, Fat List and Free Foods.

This is prepared originally by American dietetic association. ${ }^{[3]}$ For example under the Starch/Bread List one can opt for brown bread or phulka or roti or tortilla etc. depending on the availability, palatability, culture etc. Foods containing monounsaturated and polyunsaturated fats can help lower blood cholesterol levels. For example, among animal origin, cod, tuna and halibut have less total fat, saturated fat and cholesterol than do meat and poultry. Some healthy fat examples from plant source are avocados, almonds, pecans, walnuts, olives and canola, olive and peanut oils etc when taken in moderate quantity. Among various foods some examples for high in fiber include vegetables, fruits, nuts, legumes (beans, peas and lentils), whole-wheat flour and wheat bran etc. in the revised exchange list in 2008 (Wheeler et al.), dried fruits, such as blueberries, cherries, cranberries and mixed fruit, have been added and 2 tablespoons is counted as one fruit choice. ${ }^{[3]}$ A larger variety of ethnic flavours such as amaranth, bok choy, chayote and oriental radish or daikon, are now included under non starchy vegetable list. Also a new category has been added to the Milk list and its called 'Dairy-Like Foods.' Here the serving size information is given for foods such as rice drinks, smoothies and soy milk. Also list is extended to include modern food consumed worldwide such as energy/sports drinks, meal replacements/shakes and frozen yogurt etc. lastly the free food list was made to accommodate the 'Modified-Fat Foods with Carbohydrate' to alert the consumers or user of this list that remind consumers that fat-free foods, such as salad dressings, can contain a considerable amount of carbohydrate per serving.

\section{Metabolic Goals}

While making the meal plans, the calories are to be rounded off, for example, to the nearest 50-100 calories. Percentages of macronutrients have to be allotted based on metabolic goals requirements and likings of the patient. Around 45-65\% of calories from carbohydrate, 15-20\% protein and remainder by fat should be included to provide total daily energy. As dietary fibers and sugar alcohols (sorbitol, mannitol, xylitol, isomalt and hydrogenated starch hydrolysates) are incompletely digested or absorbed, amount of either is $>5$ grams per serving, we should subtract half the grams of fiber or sugar alcohol from the total carbohydrate grams in order to determine the total adjusted carbohydrate grams. ${ }^{[6]}$ Though from weight reduction point of view it may seem unnecessary, for type $1 \mathrm{DM}$ patients, it is particularly relevant in determining the insulin-to-carbohydrate ratio for patients to avoid episodes of hypoglycaemia. It essential to have a proper planning, but it is important have the attitude to sincerely implement the plan, to ensure effective glycemic control in diabetes.

\section{G. K. Pal, Nivedita Nanda}

G. K. Pal, Editor-in-Chief, IJCEP, Dept. of Physiology, JIPMER, Puducherry, India.E-mail: drgkpal@gmail.com

Nivedita Nanda, Editor, IJCEP, Dept. of Biochemistry, JIPMER, Puducherry, India. E-mail: drnnivedita@gmail.com

\section{REFERENCES}

1. The prevention and control the type-2 diabetes by changing lifestyle and dietary pattern. J Educ Health Promot. 2014;3(1):277-280.

2. Sami W, Ansari T, Butt NS, Hamid MRA. Effect of diet on type 2 diabetes mellitus: A review. Int J Health Sci (Qassim). 2017;11(2):65-71.

3. Wheeler ML, Daly A, Evert A, Franz MJ, Geil P, Holzmeister LA, et al. Choose Your Foods: Exchange Lists for Diabetes, description and guidelines for use. J Am Diet Assoc. 2008;108(5):883-8.

4. Maez L, Erickson L, Naumuk L. Diabetic education in rural areas. Rural Remote Health. 2014;14(2):2742

5. Courie R, Gaillard M, Lainas P, Hansel B, Naveau S, Dagher I, et al. Weight outcome after 2 years of a diet that excludes six processed foods: exploratory study of the "1,2,3 diet" in a moderately obese population. Diabetes Metab Syndr Obes. 2018; 12;11:345-55

6. Rivellese AA, Giacco R, Costabile G. Dietary carbohydrates for diabetics. Curr Atheroscler Rep. 2012;14(6):563-9. 